\title{
Surto de Trichophyton equinum var. equinum em eqüinos no sul do Brasil
}

\author{
Outbreak of Trichophyton equinum var. equinum in horses in the south of Brazil
}

\author{
Daniela Isabel Brayer Pereira ${ }^{1}$ Luiz Sergio Segala de Oliveira ${ }^{2}$ Andressa Bueno $^{3}$ \\ Ayrton Sydnei Cavalheiro ${ }^{3}$ Stela Elisangela Schwendler $^{3}$ Maria Isabel de Azevedo $^{3}$ \\ João Carlos Eckhardt Júnior ${ }^{3}$ Leila Cristiane Aguiar $^{3}$ Deise Flores Santurio ${ }^{4}$ \\ Janio Morais Santurio ${ }^{5}$ Sydney Hartz Alves ${ }^{5}$
}

RESUMO

\begin{abstract}
Descreve-se, neste trabalho, um surto de dermatofitose eqüina no Sul do Brasil. Cinqüienta e oito animais jovens $(55,2 \%)$ apresentavam lesões clínicas características da enfermidade. $O$ diagnóstico micológico realizado pela presença do fungo nos pêlos, associado às características macro e micromorfológicas das colônias isoladas em meio de cultivo, permitiram a classificação do dermatófito como Trichophyton equinum var. equinum.
\end{abstract}

Palavras-chave: eqüinos, dermatófitos, tinha, dermatofitose, Trichophyton equinum var. equinum.

\section{ABSTRACT}

One outbreak of dermatophytosis in horses is described in south Brazil. Fifty eight young animals (55.2\%) presented clinical signs of the disease. The mycological diagnosis was realized through the presence of fungus in the hair, associated with the macro and micromorphologic characteristics of the isolated colonies in agar also allowed the dermatophyte's classification as Trichophyton equinum var. equinum.

Key words: horses, dermatophytes, ringworm, dermatophytosis, Trichophyton equinum var. equinum.

\section{INTRODUÇÃO}

As dermatofitoses, também denominadas "tinhas", constituem-se nas micoses cutâneas mais freqüentes dos animais domésticos e humanos. São causadas por um grupo de fungos filamentosos, que, em geral, não invadem o tecido subcutâneo, ficando suas infecções restritas aos extratos queratinizados da pele e anexos. Classificam-se em três gêneros: Epidermophyton, Microsporum e Trichophyton, que incluem cerca de 40 espécies. Os animais assumem importância zoonótica, pois atuam como reservatórios dos dermatófitos considerados zoofílicos, como, por exemplo, Microsporum canis, Trichophyton verrucosum, Trichophyton mentagrophytes $e$ Trichophyton equinum (CABAÑES, 2000).

Os fungos Trichophyton equinum var. equinum e Trichophyton equinum var. autotrophicum têm como hospedeiro natural os eqüinos. A doença nesses animais foi descrita pela primeira vez por Matruchot \& Dassonoville em 1896 (GEORG et al., 1957). Desde então, alguns relatos têm sido encontrados na literatura (LONDERO et al., 1963; RIET-CORREA et al., 1984; CONNOLE \& PASCOE, 1984; CONNOLE, 1990). Embora esta espécie de dermatófito seja a principal causa da dermatofitose equiina, outras também podem estar envolvidas, assim como Microsporum equinum, Microsporum canis, Microsporum gypseum, Trichophyton mentagrophytes e Trichophyton verrucosum (GEORG et al., 1957; FRASER et al., 1993; CABAÑES, 2000).

\footnotetext{
${ }^{1}$ Programa de Pós-graduação em Ciências Veterinárias, Universidade Federal do Rio Grande do Sul (UFRGS). Correspondência para Faixa de Camobi, Km 9, prédio 20, sala 4139, 97105-900, Santa Maria, RS, Brasil. E-mail: danielabrayer@yahoo.com.br. ${ }^{2}$ Departamento de Clínica de Grandes Animais, Universidade Federal de Santa Maria (UFSM). Santa Maria, RS, Brasil.

${ }^{3}$ Curso de Graduação em Medicina Veterinária, UFSM, Santa Maria, RS, Brasil.

${ }^{4}$ Laboratório de Pesquisas Micológicas, Departamento de Microbiologia, UFSM, Santa Maria, RS, Brasil.

${ }^{5}$ Departamento de Microbiologia, UFSM, Santa Maria, RS, Brasil.
} 
A doença nos eqüinos apresenta distribuição mundial, porém é particularmente comum na Europa, nos Estados Unidos da América, no sul da América e no sul da África (GEORG et al., 1957; CUTSEM \& ROCHETTE, 1991). A Austrália é o país onde se encontra o maior número de relatos da enfermidade, porém, nesse local, a doença é causada principalmente pelo Trichophyton equinum var. autotrophicum (GEORG et al., 1957; CONNOLE, 1963; PASCOE, 1979; CONNOLE, 1990). No Brasil, a enfermidade foi diagnosticada pela primeira vez em equiinos no Estado do Rio Grande do Sul por LONDERO et al. (1963). Posteriormente, outro relato foi feito em cavalos, neste mesmo Estado, no ano de 1983 (RIETCORREA et al., 1984).

As lesões clínicas inicialmente assemelhamse à urticária, progridindo para a formação de crostas e a alopecia, sendo normalmente observadas em áreas de abrasão, principalmente no lombo, na garupa e na cabeça.

A transmissão ocorre rapidamente pelo contato direto com animais infectados ou por equipamentos contaminados. $\mathrm{O}$ estabelecimento da infecção depende de fatores do hospedeiro, como idade, imunidade, atividade fungistática das secreções cutâneas, enfermidades concomitantes e estados nutricional e hormonal (FRASER et al., 1993; PEREIRA \& MEIRELES, 2001). Geralmente, os animais jovens são mais suscetíveis e a doença costuma ser mais prevalente nos meses de outono e inverno (CONNOLE, 1963; PASCOE, 1979; CUTSEM \& ROCHETTE, 1991; PEREIRA\& MEIRELES, 2001).

O objetivo deste trabalho é descrever um surto de dermatofitose eqüina no Sul do Brasil causada por Trichophyton equinum var. equinum. A presente descrição vem corroborar com a literatura nacional, uma vez que relatos da doença em eqüinos são escassos neste país.

\section{DESCRIÇÃO DO SURTO E DIAGNÓSTICO}

Foi diagnosticado um surto de dermatofitose causado por Trichophyton equinum var. equinum em cavalos crioulos de um haras localizado no Sul do Brasil. O surto ocorreu no mês de junho do ano de 2005. De um total de 105 eqüinos, com idades entre 7-20 meses, 58 animais $(55,2 \%)$ apresentavam lesões alopécicas, circunscritas, descamativas e com intensa formação de crostas de coloração acinzentada, com evolução de aproximadamente sete dias, localizadas principalmente na cabeça e no terço anterior do pescoço (Figura 1A e 1B). Dos animais afetados, 46 estavam a campo, sendo alimentados em cochos comuns. Já os outros 12 animais encontravam-se estabulados, permanecendo em boxes individuais. Diante das características clínicas, suspeitou-se de infecção por dermatófitos, sendo coletados crostas e pêlos das bordas das lesões para maximizar o isolamento do fungo. Os referidos materiais foram utilizados para posterior diagnóstico micológico. $\mathrm{O}$ tratamento recomendado baseou-se no uso de iodophor ${ }^{a}$, diluído 1:100 ou 1:50, indicando-se banhos de aspersão semanais, durante quatro semanas. Para os animais que apresentavam lesões graves na face, também foi utilizada pomada antifúngica de cetoconazol $^{\mathrm{b}}$.

Crostas e pêlos foram submetidos ao exame direto com hidróxido de potássio a $15 \%$ como clarificador e cultivo em agar Mycobiotic ${ }^{c}$. Os tubos semeados foram incubados em estufa a $30^{\circ} \mathrm{C}$ por, no mínimo, sete dias, sendo feita a inspeção diária para avaliação das características de crescimento. No exame direto de crostas e pêlos, observou-se a presença de conídios grandes, dispostos em cadeias, formando bainhas de artroconídios em torno dos pêlos. No cultivo, evidenciou-se micélio de crescimento rápido, plano, filamentoso e algodonoso, de coloração branca a amarelada (Figura 2 A). Nas bordas das colônias, pôdese observar o crescimento de micélio submerso, o que simulava o aspecto de franjas, as quais se tornavam evidentes pela presença de pigmento amarelo brilhante, também observado no reverso das colônias. Com o envelhecimento, as colônias e o seu reverso adquiriram coloração vermelho-amarronzada (Figura 2 B). A micromorfologia dos isolados utilizando azul de algodão demonstrou grande quantidade de microconídios piriformes, sésseis ou brotando ao longo das hifas (Figura 3). Macroconídios foram raramente observados e, quando presentes, apresentavam paredes lisas, finas e de tamanhos variados. Em culturas mais velhas, houve a formação de numerosos clamidoconídios. Os testes de urease, os quais foram positivos dentro de cinco dias, de perfuração em pêlo, negativo após 21 dias, e a necessidade de ácido nicotínico para crescimento em meios livres de vitaminas, juntamente com as características macro e micromorfológicas das colônias isoladas, permitiram caracterizar o dermatófito como Trichophyton equinum var. equinum. A classificação e a identificação basearam-se nas descrições de GEORG et al. (1957) e LONDERO et al. (1963).

\section{DISCUSSÃO}

Em vários países, a dermatofitose eqüina é relatada como sendo causada por Trichophyton equinum, conforme descrições de VRIES \& JITTA 


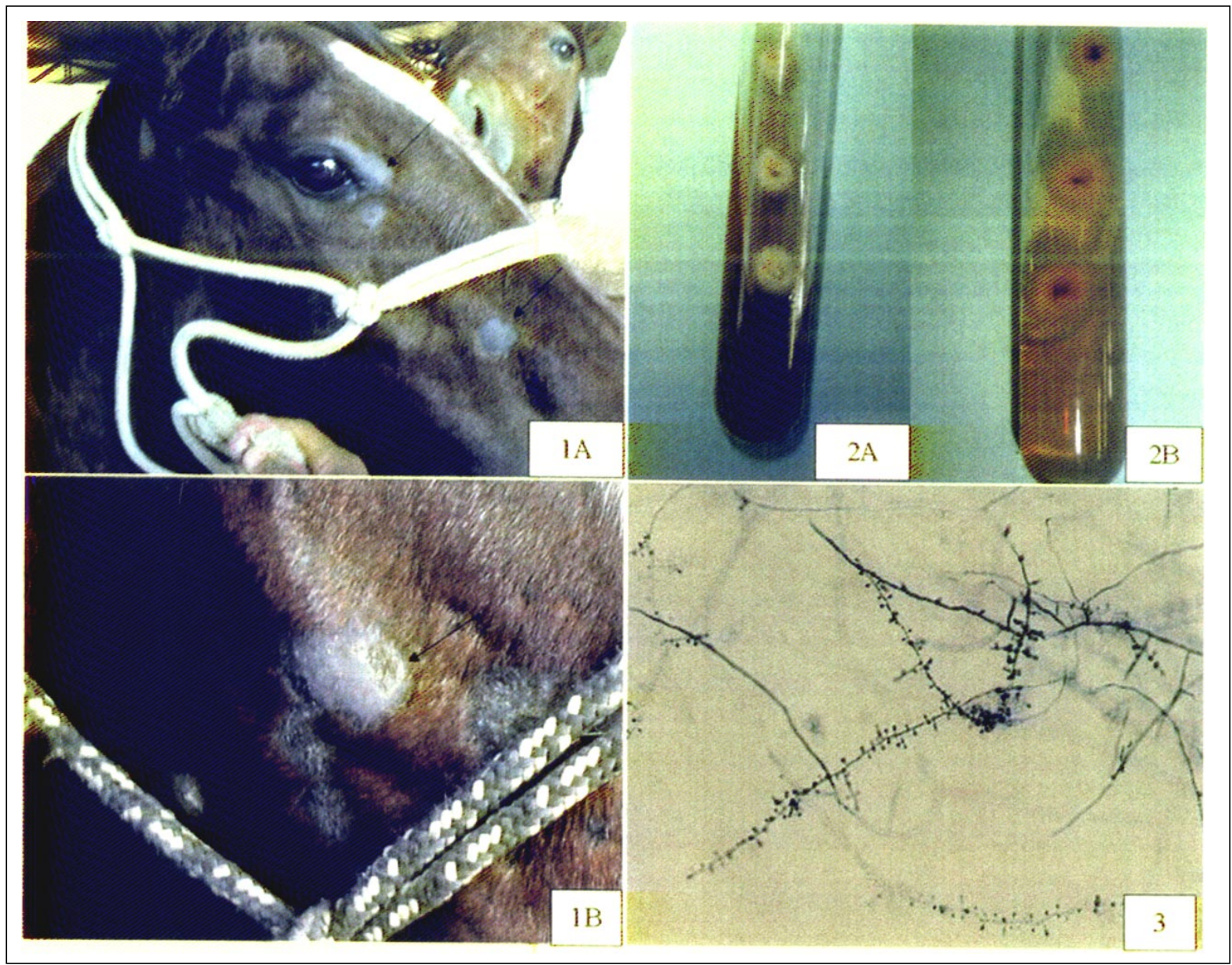

Figuras 1A e 1B - Lesões alopécicas, circunscritas e acinzentadas disseminadas na face de um eqüino (setas). Evolução de sete dias. Figura 2A: Cultivo de sete dias de Trichophyton equinum var equinum (agar Mycobiotic). Verso da colônia. Micélio filamentoso branco e amarelo. Figura 2B: reverso da colônia. Presença de pigmento amarelo brilhante tornando-se avermelhado. Figura 3: Trichophyton equinum var. equinum: Microconídios piriformes sésseis e brotando ao longo das hifas (Azul de algodão, $40 \mathrm{X}$ ).

(1973), STROSS et al. (1978), MAHMOUD (1995), CABAÑES et al. (1997), MORETTI et al. (1998). Na Austrália, país onde existe o maior número de relatos da doença, a enfermidade ocorre principalmente pela infecção de Trichophyton equinum var. autotrophicum, que se diferencia do Trichophyton equinum var. equinum pela sua capacidade de crescer sem necessidade de ácido nicotínico em meios livres de vitaminas (CONNOLE \& PASCOE, 1984). Já em alguns países como Jordânia e Iran, o principal dermatófito envolvido é o Microsporum equinum, com taxas de $40 \%$ (AL-ANI et al, 2002) e 53\% (KHROSRAVI $\&$ MAHMOUDI, 2003), respectivamente.

No presente trabalho, a identificação de Trichophyton equinum var. equinum foi baseada nas características macro e micromorfológicas, no teste de urease, no teste de perfuração em pêlo e na necessidade de ácido nicotínico para crescimento em meios livres de vitaminas, estando essas características de acordo com as já anteriormente descritas por GEORG et al. (1957), o que permitiu a sua diferenciação do Trichophytum equinum var. autotrophycum e do Microsporum equinum.

As lesões clínicas observadas nos animais afetados do presente relato foram similares àquelas descritas por GEORG et al. (1957), LONDERO et al. (1963), VRIES \& JITTA(1973) e CONNOLE \& PASCOE (1984).

O surto descrito neste estudo, afetando 58 eqüinos jovens $(55,2 \%)$ de um mesmo haras, foi observado no mês de junho, estando de acordo com PEREIRA \& MEIRELES (2001), que afirmam que as maiores freqüências da doença são observadas em animais jovens, principalmente nos meses de outono e inverno. A maior freqüência nesta época do ano pode ser explicada porque há o aumento do crescimento dos 
pêlos dos animais, que, associado às trocas metabólicas com desprendimento de calor, favorecem um microclima, ideal para o crescimento do dermatófito. No caso descrito por LONDERO et al. (1963), 58,6\% dos animais estavam infectados pelo fungo e, na descrição de RIET-CORREA et al. (1984), a frequiência atingiu 47,37\% dos animais, caracterizando frequiências relativamente altas da enfermidade.

Em estudos realizados na Austrália, observouse que, nos meses de verão e outono, coincidentes com os períodos de alta umidade, $76,5 \%$ dos eqüinos com idade inferior a 4 anos encontravam-se infectados (PASCOE, 1979). Descrições da doença em outros países também citam altas freqüências em animais jovens (STROSS et al., 1978; MAHMOUD, 1995).

$\mathrm{O}$ fato de ter-se encontrado 46 animais de campo com lesões na cabeça e no terço anterior do pescoço pode ser explicado por esses animais serem alimentados em cochos comuns, favorecendo o contato direto e a conseqüente disseminação do dermatófito. Já nos animais que se encontravam estabulados, a disseminação ocorreu pelo uso comunitário de equipamentos como escovas, mantas e arreios. Entre os animais, a doença pode ser transmitida por contato direto ou indireto com fômites contaminados, conforme citado por CUTSEM \& ROCHETTE (1991); FRASER et al (1993); PEREIRA \& MEIRELES (2001). A velocidade com que essa doença se transmite é muito rápida, principalmente quando se utilizam equipamentos em comum, os quais podem veicular artroconídios do fungo por até 12 meses (PASCOE, 1979).

O tratamento com iodophor foi eficaz, o que também foi observado por PASCOE (1984), que, ao testar 12 diferentes medicamentos para dermatofitose eqüina, observou que compostos de iodo-povidine, além de apresentarem resultados satisfatórios, eram de fácil aplicação para tratamento de grandes áreas de lesões e não apresentavam efeitos adversos.

A dermatofitose eqüina não implica conseqüências pela mortalidade, pois esta é praticamente nula, mas pelo fato que eqüinos que apresentam lesões clínicas são impedidos de participar de exposições, feiras e remates devido ao caráter contagioso da enfermidade. Além disso, como se trata de uma zoonose, tratadores e outros indivíduos que tenham contato com esses animais podem vir a contrair a doença, o que tem sido bem caracterizado em relatos de casos de dermatofitose humana causada pelo Trichophytum equinum descritos por TAKATORI \& ICHIGO(1985); HUOVINEN(1998)eAMOR etal. (2001).

\section{CONCLUSÃO}

As características macro e micromorfológicas do fungo isolado permitiram a identificação de Trichophyton equinum var. equinum, demonstrando a presença desta espécie de dermatófito como agente de dermatofitose em eqüinos no Rio Grande do Sul.

\section{FONTES DE AQUISIÇÃO}

a- Laboratório Pfizer

b- Laboratório Jansen Cilag

c- Biobrás Diagnósticos

\section{REFERÊNCIAS}

AL-ANI, F.K. et al. Ringworm infection in catlle and horses in Jordan. Acta Veterinaria Brno, v.71, p.55-60, 2002.

AMOR, E. et al. Terbinafine treatment of Trichophytum equinum infection in a child. Clinical and Experimental Dermatology, v.26, p.276-278, 2001.

CABAÑES, F.J. et al. Dermatophytes isolated from domestic animals in Barcelona, Spain. Mycopathologia, v.137, p.107$113,1997$.

CABAÑES, F.J. Dermatophytes in domestic animals. Revista Iberoamericana de Micologia, v.17, p.104-108, 2000.

CONNOLE, M.D. A review of dermatomycoses of animals in Australia. Australian Veterinary Journal, v.39, p.130-134, 1963.

CONNOLE, M.D.; PASCOE, R.R. Recognition of Trichophyton equinum var. equinum infection of horses. Australian Veterinary Journal, v.61, n.3, p.94, 1984.

CONNOLE, M.D. Review of animal mycoses in Australia. Mycopathologia, v.111, p.133-164, 1990.

CUtSem, J.V.; ROChetTe, F. Mycoses in domestic animals. Bélgium: Janssen Research Fundation, 1991. 226p.

FRASER, et al. El manual Merck de veterinaria. Barcelona: Oceano/Centrum, 1993. 2092p.

GEORG, L.K. et al. Equine ringworm with special reference to Trichophyton equinum. American Journal Veterinary Research, p.798-810, 1957.

HUOVINEN, S. et al. Human onychomycosis caused by Trichophytum equinum transmitted from racehorse. British Journal of Dermatology, v.138, p.1082-1084, 1998.

KHROSRAVI, A.R.; MAHMOUDI, M. Dermatophytes isolated from domestic animals in Iran. Mycoses, v.46, p.222-225, 2003.

LONDERO, A.T. et al. An epizootic of Trichophyton equinum infecction on horses in Brazil. Sabouraudia, v.3, n.1, p.14$15,1963$. 
MAHMOUD, A.L. Dermatophytes and other keratinophilic fungi causing ringworm of horses. Folia Microbiol, Praha, v.40, n.3, p.293-296, 1995.

MORETTI, A. et al. Epidemiological aspects of dermatophyte infections in horses and catlle. Zentralbl Veterinarmed B, v.45, n.4, p.205-208, 1998.

PASCOE, R.R. The epidemiology of ringworm in racehorses caused by Trichophyton equinum var. autotrophicum Australian Veterinary Journal, v.55, p.403-407, 1979.

PASCOE, R.R. Experimental medication of equine ringworm due to Trichophytum equinum var. autotrophicum. Australian Veterinary Journal, v.61, n.7, p.231-235, 1984.

PEREIRA, D.B.; MEIRELES, M.C.A. Doenças causadas por fungos e oomycetos. In: RIET-CORREA, F. et al. Doenças de ruminantes e eqüinos. São Paulo: Varela, 2001. cap.4, p.367-383.

RIET-CORREA, F. et al. Laboratório regional de diagnóstico. Doenças Diagnosticadas no ano 1983. Pelotas: UFPel, 1984. p.20.

STROSS, K. et al. Occurrence of dermatophytosis of horses caused by the fungus Trichophyton equinum. Veterinary Medicine, v.23, n.3, p.175-184, 1978

TAKATORI, K.; ICHIGO, S. Human dermatophytosis caused by Trichophytum equinum. Mycopathologia, v.90, p.15-19, 1985.

VRIES, DE G.A.; JITTA, J.C.R. An epizootic in horses in the Netherlands caused by Trichophytum equinum var. equinum. Sabouraudia, v.11, p.137-139, 1973. 